\title{
Perceived barriers to healthy eating: a systematic review
}

\author{
J. Lara ${ }^{1,2}$, V. Yong ${ }^{1}$ and J.C. Mathers ${ }^{1}$ \\ ${ }^{1}$ Human Nutrition Research Centre, Institute of Cellular Medicine; Newcastle University. Biomedical Research \\ Building, Campus for Ageing and Vitality. Newcastle Upon Tyne, NE4 5PL, UK and ${ }^{2}$ Department of Applied \\ Sciences, Faculty of Health and Life Sciences, Northumbria University, Ellison Building Room A324, Newcastle upon \\ Tyne, NE1 8ST, UK
}

Strong evidence indicates that lifestyle behaviours including poor diet, smoking, low physical activity, high alcohol consumption, or high body mass index (BMI), are strongly associated with health in adults. Together, these behaviours are associated with four-fold increases in total mortality in men and women and make a substantial contribution to the global burden of disability ${ }^{1}$. Identifying and addressing perceived barriers to healthy eating (PBHE) is a critical step in developing effective dietary interventions to promote health in adulthood ${ }^{2,3}$. A critical analysis of the evidence in this area will be valuable in developing interventions targeting healthier eating patterns.

We undertook a systematic review of studies evaluating PBHE among adults $>18$ years. The protocol for the review was registered with PROSPERO International prospective register of systematic reviews (CRD42015027946). A search of three databases 1) OVID Medline 2) Embase and 3) Scopus, until October 2015 produced 2091 papers. After abstract and full paper screening, 92 papers published between 1996 and 2015 met our inclusion criteria. Most were cross-sectional studies $(n=88)$, while only 4 reported intervention studies. Participants in these studies were adults mean age for studies ranging from 23.8 years to 74.8 years. A total of 215 terms related to PBHE were identified. The number of PBHE in individual studies ranged from 1 to 21 . A card sorting task involving 3 pairs of volunteers was undertaken to develop logical categories of PBHE.

\begin{tabular}{|c|c|c|c|}
\hline Origin of studies & $\begin{array}{l}\text { Methods investigating PBHE } \\
\text { (number of studies) }\end{array}$ & $\begin{array}{l}\text { PBHE categories showing the } \\
\text { greatest agreement between card sorters }\end{array}$ & $\begin{array}{l}\text { Top } 5 \text { most commonly } \\
\text { reported barriers }\end{array}$ \\
\hline United States $42 \%$ & Surveys (31) & 1. Education & 1. Time \\
\hline United Kingdom $15 \%$ & Focus groups (28) & 2. Personal & 2. Cost /price \\
\hline Australia $9 \%$ & Interviews (23) & 3. Culture & 3. Cooking skills \\
\hline \multirow{4}{*}{ Other countries $34 \%$} & Other methods (10) & 4. Environment & 4. Knowledge \\
\hline & & 5. Economic & 5. Taste /pleasant \\
\hline & & 6. Time & \\
\hline & & 7. External & \\
\hline
\end{tabular}

Few studies investigated facilitators of healthy eating helping to overcome these PBHE; this is an area that requires further investigation. The results of this study have important implications for researchers interested in behaviour change. The PBHE identified can inform the design of tailored, or personalised, interventions for adults ${ }^{4}$.

1. Khaw KT, Wareham N, Bingham S, Welch A, Luben R, Day N. PLoS Med 2008; 5:E152.

2. Mathers JC. Br J Nutr 2015; 113 Suppl:S18-22.

3. Lara J, McCrum LA, Mathers JC. Maturitas 2014; 79:292-298.

4. Celis-Morales C, Lara J, Mathers JC. Proc Nutr Soc 2015; 74: 130-138. 